\title{
Synthesis of Sulfonamide Derived from New Tetrahydroquinazoline Derivatives via Intramolecular cyclization
}

\author{
Biswadip Banerji, Moumita Chatterjee, Suvankar Bera and Mohd \\ Usman Mohd Siddique \\ Department of Chemistry; CSIR-Indian Institute of Chemical Biology; 4, Raja S.C. Mullick Road, Kolkata, \\ India
}

Correspondence should be addressed to: Biswadip Banerji; biswadip@iicb.res.in

Received date: 20 September 2013; Accepted date: 21 November 2013; Published date: 30 January 2014

Academic Editor: Nilo Zanatta

Copyright (C) 2014. Biswadip Banerji, Moumita Chatterjee, Suvankar Bera and Mohd Usman Mohd Siddique. Distributed under Creative Commons CC-BY 3.0

\begin{abstract}
Tetrahydroquinazoline and sulfonamide are two very important class of heterocycles, showing a wide spectrum of biological and biochemical activities on the one hand. On the other hand, there is a growing demand of new pharmacophore, which can truly replace the old resistant marketed drug. Consequently we are interested in synthesizing new molecules containing hybrid scaffold having both the tetrahydroquinazoline and sulfonamide moiety, as both are very active pharmacophore. In the present communication, sulphonamide derived tetrahydroquinazoline heterocyclic rings have been synthesized by using intramolecular cyclization reaction. Using this methodology, a small library of ten compounds has been synthesized. The structure of the compound was unambiguously resolved by the single X-ray crystal structure of some of the molecules. A highly substituted heterocycles can be easily synthesized by this methodology. Moreover these new sulfonamide derived tetrahydroquinazoline compounds may act as very potent inhibitors of different enzymes or proteins, responsible for many diseases.
\end{abstract}

Keywords: Heterocycles, Quinazoline, Sulfonamide, Electronic effect

\section{Introduction}

Heterocyclic compounds are one of the most important classes of organic compounds that have a wide range of biological activities: antibacterial, antifungal, and several other biological activities. Amongst these are sulfur and nitrogen containing heterocycles, are especially important. Consequently, the

Cite this Article as: Biswadip Banerji, Moumita Chatterjee, Suvankar Bera and Mohd Usman Mohd Siddique (2014), "Synthesis of Sulfonamide Derived from New Tetrahydroquinazoline Derivatives via Intramolecular cyclization", JMED Research, Vol. 2014 (2014), Article ID 464776, DOI: 10.5171/2014. 464776 
development of new methodologies useful for the synthesis of new heterocycle templates has gained much attention in both the academic and the industrial communities. Thus there is a growing need for new scaffolds which can act better, compared to the previous known drugs.
Quinazoline is one such heterocycles which may contain a versatile pharmacophore responsible for many biological activities. It possesses a wide spectrum of bioactivities as antimicrobial, bronchodilator, antihistaminic, anti-inflammatory, angiotensin (II) receptor antagonist, antiherpes, anti-tubercular, antiinsecticidal and cardiovascular agent etc.<smiles>COc1cc2nc(N3CCN(C(=O)c4ccco4)CC3)nc(N)c2cc1OC</smiles>

Prazosin: Sympatholytic drug<smiles>COc1cc2ncnc(Nc3ccc(F)c(Cl)c3)c2cc1OCCCN1CCOCC1</smiles>

Gefitinib: EGFR inhibitor<smiles>Cc1ccc2c(-c3ccccc3)nc(=O)n(C(C)C)c2c1</smiles>

Proquazone: Anti-inflammatory drug<smiles>COc1cc(NCc2ccc3nc(N)nc(N)c3c2C)cc(OC)c1OC</smiles>

Trimetrexate: Dihydrofolate reductase inhibitor

Figure1: Biologically active molecules with Quinazoline scaffold

Among the different bioactive molecules, sulfonamide group containing heterocycles are present at large. Owing to its wide application as antibiotics, anti-carbonic anhydrase, diuretic, hypoglycemic, anti thyroid and anticancer agent. Researchers across the world are also interested in synthesizing new sulfonamide derived heterocyclic scaffolds. However the rapid emergence of sulfonamide resistant organisms restricted its clinical application. It constantly needs new molecules to replace the old one with new scaffolds and new pathway of mechanism of action in each case.
Moreover, sometimes it becomes necessary to treat an infection with potentially toxic drugs against those organisms which are resistant to all approved antibiotics. Consequently, there is an ever-growing demand for more effective antibacterial agents against antibiotic resistant bacteria pathogens. Therefore, research efforts are focused to make new antibiotics, those which can kill the bacteria by a new pathway. Considering the potential of quinazoline and sulfonamide moiety separately, we were interested in the synthesis of new hybrid heterocyclic scaffold containing both the quinazoline and sulfonamide moiety. 


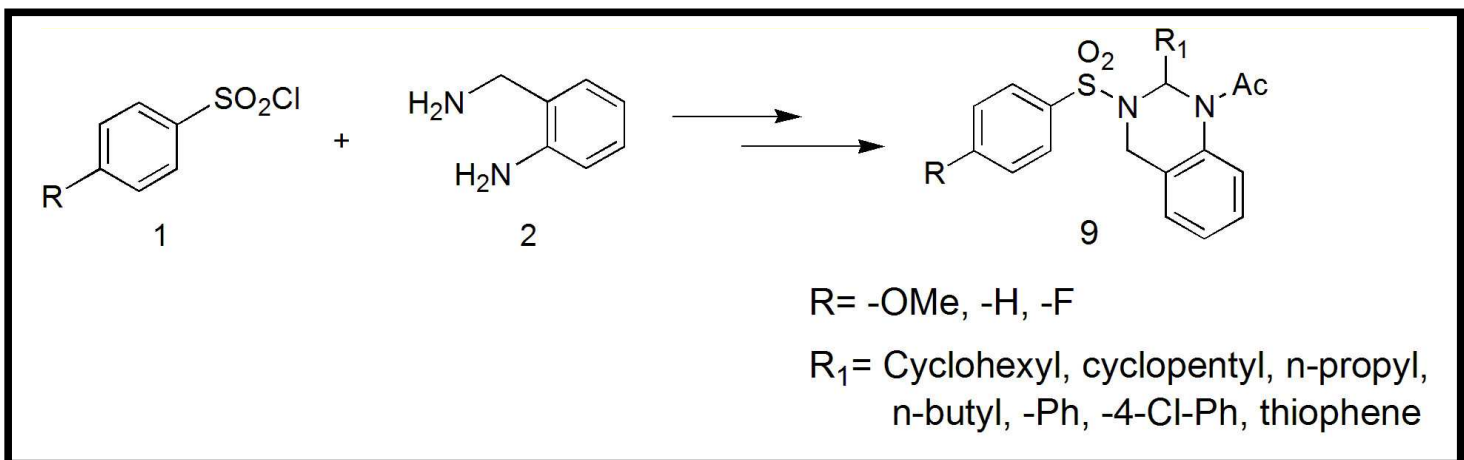

Schema1: Synthetic Route to the " $N^{1}$-acyl- $N^{2}$-sulfono Quinazoline

The following scheme depicts the general synthetic route for the synthesis of aryl sulfonyl group substituted tetrahydroquinazoline (Scheme: 1). To our knowledge, this is the first example of sulfonyl and acyl group substituted tetrahydroquinazoline (" $N{ }^{1}$-acyl- $N^{2}$-sulfono Quinazoline") scaffold. Owing to its importance, we have planned to synthesize a small library of these molecules using the above methodology. It is believed that this methodology will open up new easy access to this class of molecules.

\section{Result and Discussion}

The synthesis of $N^{1}$-acyl- $N^{2}$-sulfono Quinazoline scaffold library started by condensing an aromatic sulfonyl chloride (1) and a 2-aminobenzyl amine (2). The free amine present in the generated sulfonamide (3) undergoes Schiff base (5) formation with the aldehydes (4) in the next step. The Schiff base was then converted to the $\mathrm{N}$-acylinium ion (6) by treating them with acetyl chloride, which in situ undergoes intramolecular cyclization under basic condition as shown in scheme 2 , to produce the highly substituted tetrahydroquinazoline in moderate to good yield. It is worthy to mention here that we are able to confirm the structure of the $N^{1}$ acyl- $N^{2}$-sulfono Quinazoline derivatives unambiguously by the single X-ray crystal structure of compound 9a and 9j (Fig: 2). The crystal structure also corroborates the mechanistic pathway as proposed in scheme 2. Based on this methodology we have successfully synthesized a small library of 10 different quinazoline molecules in good to moderate yields. The rate of cyclization reaction, as is observed, has tremendous effect on the electronic nature of the Rgroups of aldehydes and the substituents effect on the aromatic rings of the sulfonamide part. 


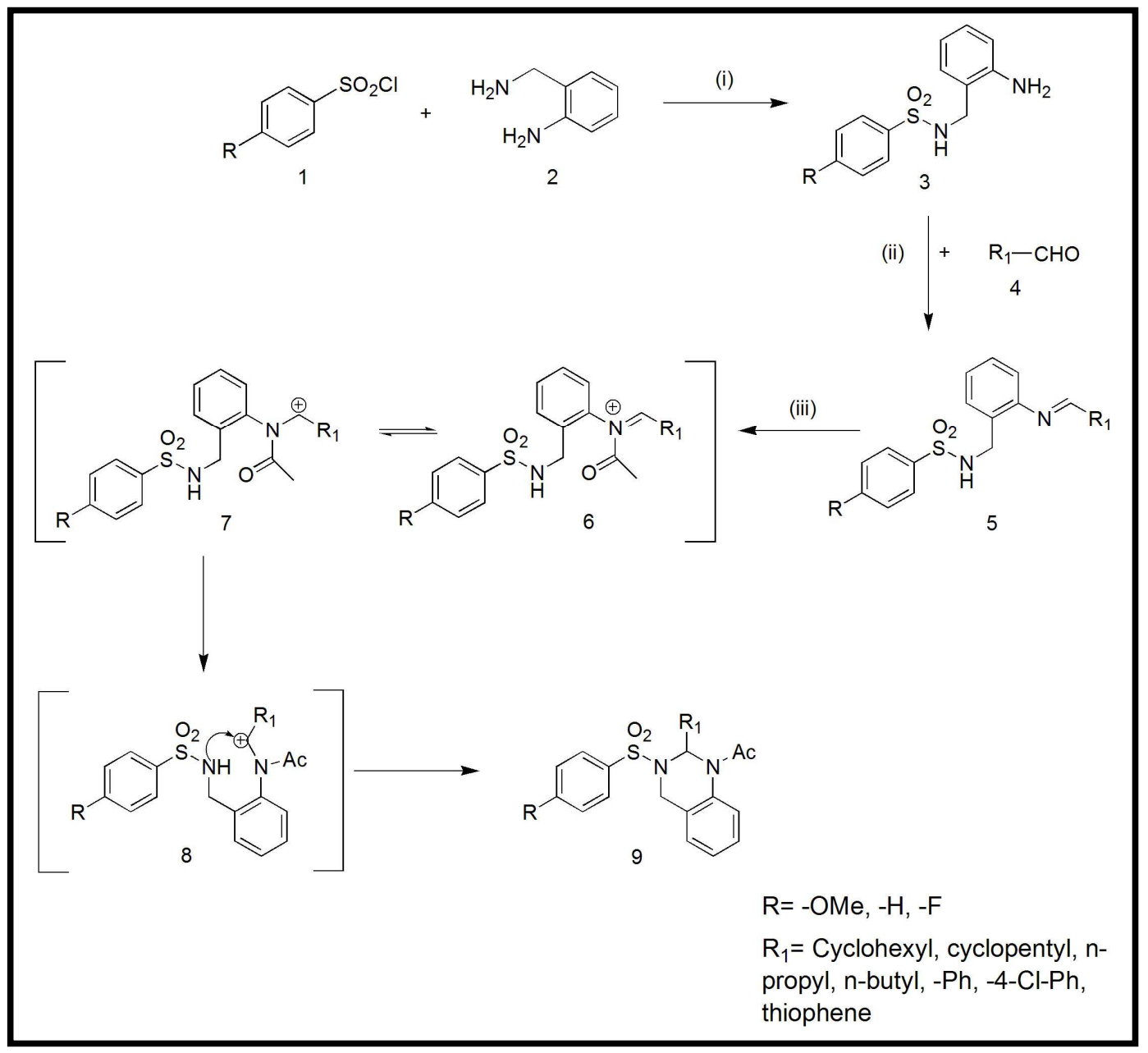

Schema 2: General scheme for the synthesis of $N^{1}$-acyl- $N^{2}$-sulfono Quinazoline

Reagents and Conditions:(i) NaOAc, aq EtOH (ii) $\mathrm{EtOH}$, glacial AcOH (iii) 2,6-Leutidine, $\mathrm{AcCl}, \mathrm{THF} / \mathrm{Et}_{2} \mathrm{O}, 0^{0} \mathrm{C}$ to r.t.

Remarkably the cyclization worked well with aliphatic aldehydes and was quite sluggish with aromatic ones. Therefore the nature of aldehydes provided sensible impact on the final cyclization reaction. Actually the cyclization is dependent on the electrophilicity of the iminium carbocataion i.e. greater eletrophilicity facilitates the reaction. 
Table1: Ten new Quinazoline derivatives made from aliphatic and aromatic aldehydes

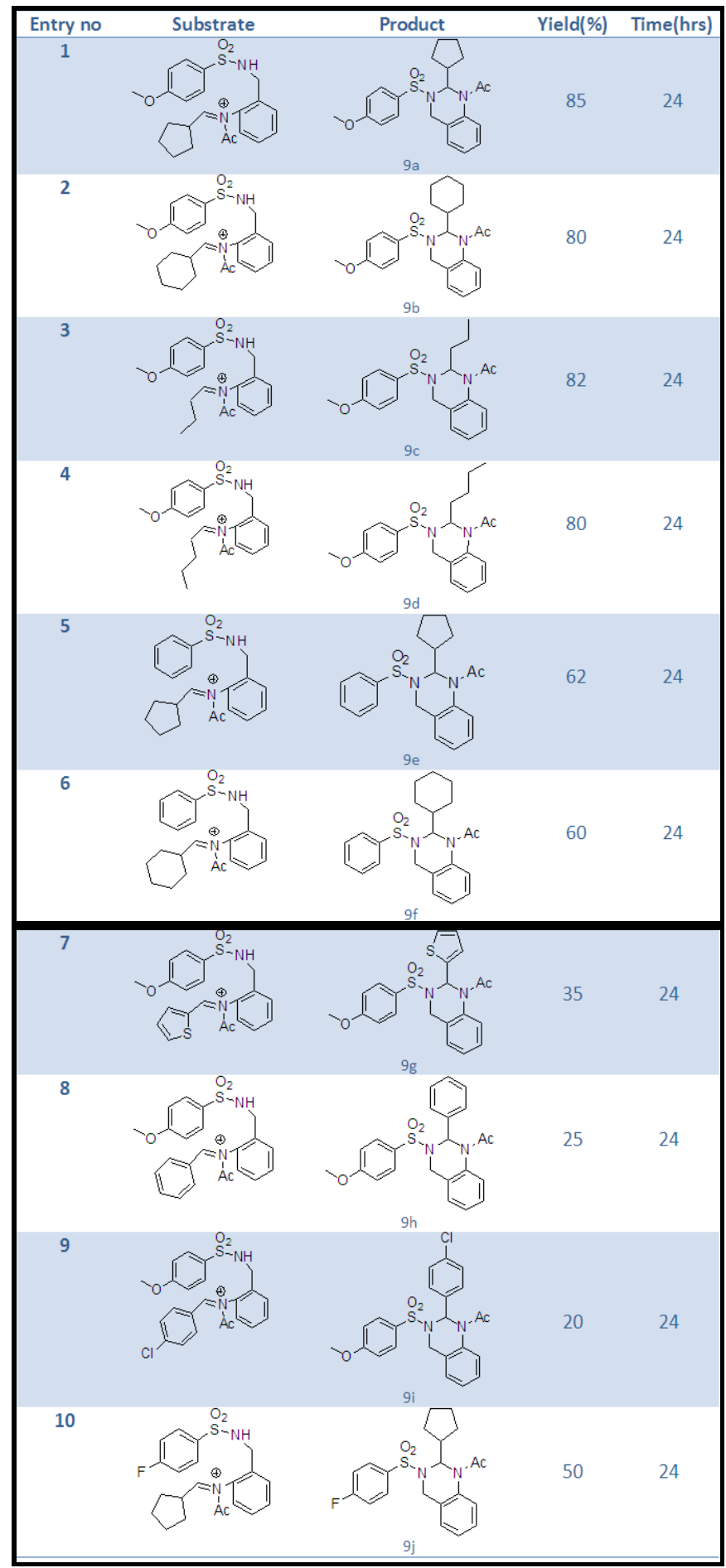

Biswadip Banerji, Moumita Chatterjee, Suvankar Bera and Mohd Usman Mohd Siddique (2014), JMED Research, DOI: 10.5171/2014. 464776 
For aromatic schiff's base, +R effect of aldehydic aromatic ring decreases the electrophilicity of the carbocation and exerts detrimental effect on the final cyclization (Table 1, 9h, 9i), whereas $+\mathrm{I}$ effect (which is less stronger than $+R$ ), of the aliphatic group of aldehydes showed no such effect towards the rate of cyclization (Table 1, 9a-f, 9j).

Apart from the stability of iminium carbocation, nucleophilicity of the sulfonamide moiety also influences the outcome of the cyclization significantly. In case of product 9a, cyclization is facilitated by the electron donating -OMe group in the aryl ring of the sulfonamide part which stabilizes the iminium carbocation and gave $85 \%$ yield of the desired cyclic product, whereas the yield drops to $62 \%$ for product $9 \mathrm{e}$ due to the absences of $-\mathrm{OMe}$ group. Similar trend was observed for compound $9 \mathrm{~b}$ and 9f. Again for product 9j, due to the presence of deactivating $-\mathrm{F}$ group, $50 \%$ yield was obtained. Hence, this reaction is substituent tolerable and can be applicable to a wide variety of substrates.

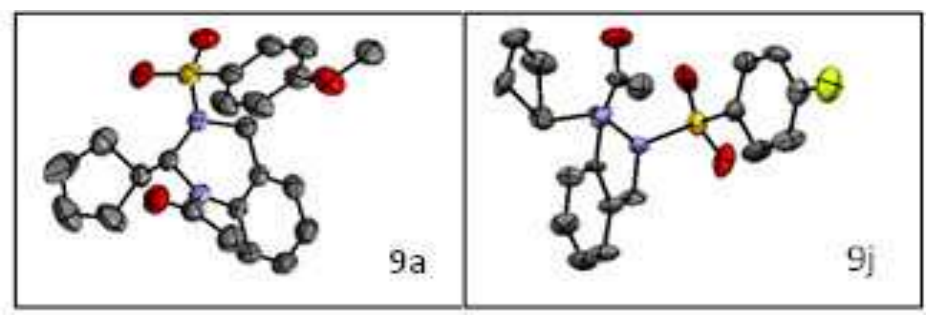

Fig2: Single X-ray crystal structures of compounds 9a and 9j

\section{Conclusion}

We have successfully synthesized $10 N^{1}$-acyl$N^{2}$-sulfono Quinazoline derivatives via $\mathrm{N}$ acylinium intermediate and also achieved the cyclization with electron deficient sulfonamide as nucleophile. So, this general strategy for pursuing highly active electrophiles to facilitate the intramolecular nucleophilic attack becomes a basis for synthesizing biologically active more functionalized quinazoline derivatives. Higher yield of the cyclized product was observed in case of activated aromatic ring. Since these $N^{1}$-acyl- $N^{2}$-sulfono Quinazolines obtained as racemic mixture, the streoselective version of this cyclization is our next target. The library of these new heterocyclic scaffold and the compounds therefore may act as good inhibitors of different enzymes or proteins. Biological studies of these molecules are going on in the present time in our laboratory, and the results will be published in the future.

\section{Experimental Section}

\section{General Procedure for the Final Cyclization}

To a stirred solution of schiff base (5) (1 mmole) dissolved in THF or diethylether (as per requirement) at $0^{0} \mathrm{C}, 2,6$-leutidine (1 mmole) was added followed by acetyl chloride (1mmole) addition under nitrogen atmosphere (scheme 2). The reaction was continued for $24 \mathrm{hrs}$. The solvent was evaporated to concentrate the reaction mass and the residue obtained was purified by column chromatography (hexane/ethyl acetate) to afford pure cyclized product.

\section{1-(2-Cyclopentyl-3-(4-methoxy-} benzenesulfonyl)-3,4-dihydro-2Hquinazolin-1-yl)-ethanone (9a): White solid, $\mathrm{R}_{\mathrm{f}} \approx 0.40$ (petroleum ether, ethyl acetate 3:2), Melting point $155^{\circ} \mathrm{C},{ }^{1} \mathrm{H}$ NMR $\left(600 \mathrm{MHz}, \mathrm{CDCl}_{3}\right.$ ): $\delta$ (in ppm) 1.327-1.490 $(4 \mathrm{H}, \mathrm{m}), 1.641-1.710(4 \mathrm{H}, \mathrm{m}), 1.833(3 \mathrm{H}, \mathrm{s})$, $1.918(1 \mathrm{H}, \mathrm{m}), 3.807(3 \mathrm{H}, \mathrm{s}), 4.525(1 \mathrm{H}, \mathrm{d}, J=$ 17.4), $4.780(1 \mathrm{H}, \mathrm{d}, J=18), 6.325(1 \mathrm{H}, \mathrm{d}, J=$ 10.2), 6.873-6.912 (3H, m), 7.146-7.207 (3H, m), 7.727 (2H, d, $J=7.2) ;{ }^{13} \mathrm{C}$ NMR $(150 \mathrm{MHz}$, 
$\mathrm{CDCl}_{3}$ ): $\delta$ (in ppm) 22.61, 25.08, 25.19, 27.83, 28.84, 41.30, 43.49, 55.55, 66.46, 114.07, $114.54,124.45,125.18,125.50,126.43$, $127.18,129.33,129.88,130.53,135.18$, 162.89, 169.01; HRMS (EI+): $\mathrm{m} / \mathrm{z}$ Calcd for $\mathrm{C}_{22} \mathrm{H}_{26} \mathrm{~N}_{2} \mathrm{O}_{4} \mathrm{~S}$ (M)+ 414.1613, Found: $\mathrm{m} / \mathrm{z}$ 414.16132. IR (in $\mathrm{cm}^{-1}$ ) 565, 615.56, 671.30, 748.91, 804.99, 835.42, 962.61, 1020.74, 1091.86, 1160.93, 1264.53, 1322.65, $1339.75,1378.73,1499.43,1596.53$, 1654.66, 2867.10, 2962.84, 3008.32, 3445.52 .

\section{1-(2-Cyclohexyl-3-(4-methoxy- benzenesulfonyl)-3,4-dihydro-2H- quinazolin-1-yl)-ethanone (9b): White} solid, $\mathrm{R}_{\mathrm{f}} \approx 0.50$ (petroleum ether, ethylacetate $3: 2$ ), Melting point $120^{\circ} \mathrm{C},{ }^{1} \mathrm{H}$ NMR (600 MHz, $\mathrm{CDCl}_{3}$ ): $\delta$ (in ppm) 0.86-0.94 $(1 \mathrm{H}, \mathrm{m}), 1.05-1.17(3 \mathrm{H}, \mathrm{m}), 1.38-1.41(1 \mathrm{H}, \mathrm{m})$, $1.55(1 \mathrm{H}, \mathrm{d}, J=9.2), 1.65(3 \mathrm{H}, \mathrm{s}), 1.77(1 \mathrm{H}, \mathrm{d}, J$ = 18), $1.84(3 \mathrm{H}, \mathrm{s}), 1.93-1.94(1 \mathrm{H}, \mathrm{m}), 3.80$ $(3 \mathrm{H}, \mathrm{s}), 4.46(1 \mathrm{H}, \mathrm{d}, J=18), 4.76(1 \mathrm{H}, \mathrm{d}, J=$ 18), 6.27-6.28 $(1 \mathrm{H}, \mathrm{m}), 6.86-6.90(3 \mathrm{H}, \mathrm{m})$ 7.14-7.20 (3H, m), $7.71(2 \mathrm{H}, \mathrm{d}, J=6) ;{ }^{13} \mathrm{C} \mathrm{NMR}$ (150 $\mathrm{MHz}_{\mathrm{CDCl}}$ ): $\delta$ (in ppm) 22.62, 25.46, $25.91,27.99,28.88,29.67,37.96,43.29$, $55.55,66.89,114.08,114.39,124.43,125.05$, $125.52,126.48,127.16,129.35,129.84$, 130.63, 134.93, 162.88, 169.27; HRMS (EI+): $\mathrm{m} / \mathrm{z}$ Calcd for $\mathrm{C}_{23} \mathrm{H}_{28} \mathrm{~N}_{2} \mathrm{O}_{4} \mathrm{~S}(\mathrm{M})+428.17698$, Found: $\mathrm{m} / \mathrm{z}$ 428.17697. IR (in $\mathrm{cm}^{-1}$ ) 561.54, 617.27, 671.30, 746.86, 804.99, 833.02, 964.66, 1020.74, 1039.89, 1147.93, 1165.03, $1264.18, \quad 1322.65,1378.39,1462.50$, $1447.45, \quad 1499.43, \quad 1585.59, \quad 1596.19$, 1654.66, 2848.75, 2923.52, 3435.03.

\section{1-[3-(4-methoxy-benzenesulfonyl)-2-} propyl-3,4-dihydro-2H-quinazolin-1-yl)ethanone (9c): White solid, $\mathrm{R}_{\mathrm{f}} \approx 0.50$ (petroleum ether, ethyl acetate 3:2), Melting point $130^{\circ} \mathrm{C},{ }^{1} \mathrm{H}$ NMR $\left(600 \mathrm{MHz}, \mathrm{CDCl}_{3}\right): \delta$ (in ppm) 0.86-0.89 (3H, m), 1.39-1.44 $(2 \mathrm{H}, \mathrm{m})$, $1.59(3 \mathrm{H}, \mathrm{s}), 1.87-1.88(2 \mathrm{H}, \mathrm{m}), 3.81(3 \mathrm{H}, \mathrm{s})$, $4.49(1 \mathrm{H}, \mathrm{d}, J=12), 4.63(1 \mathrm{H}, \mathrm{d}, J=18), 6.63-$ $6.66(1 \mathrm{H}, \mathrm{m}), 6.89(2 \mathrm{H}, \mathrm{d}, J=6), 6.93-6.94$ $(1 \mathrm{H}, \mathrm{m}), 7.15-7.16(2 \mathrm{H}, \mathrm{m}), 7.21(1 \mathrm{H}, \mathrm{t}, J=6)$, $7.74(2 \mathrm{H}, \mathrm{d}, J=12)$; ${ }^{13} \mathrm{C}$ NMR $(150 \mathrm{MHz}$, $\mathrm{CDCl}_{3}$ ): $\delta$ (in ppm) 13.42, 18.18, 22.66, 34.78, $43.40,55.56,62.68,113.51,114.16,124.65$, $125.42,125.67,126.36,126.58,127.33$,
129.45, 129.79, 130.27, 162.99, 168.91; HRMS (EI+): $\mathrm{m} / \mathrm{z}$ Calcd for $\mathrm{C}_{20} \mathrm{H}_{24} \mathrm{~N}_{2} \mathrm{O}_{4} \mathrm{~S}(\mathrm{M})^{+}$ 388.14568, Found: $\mathrm{m} / \mathrm{z} 388.14554$. IR (in $\mathrm{cm}^{-}$ 1) 557.10, 615.22, 632.66, 673.35, 722.93, 753.36, 759.85, 802.94, 835.08, 928.08, 958.17, 1027.23, 1037.83, 1089.46, 1113.40, 1147.93, 1158.53, 1262.13, 1309.66, 1328.81, 1372.23, 1460.45, 1497.03, $1585.59, \quad 1596.19, \quad 1652.26,1663.20$, 2866.08, 2923.52, 2975.49, 3435.03.

\section{1-(3-benzenesulf onyl-2-cyclopentyl-3,4-} dihydro-2H-quinazolin-1-yl)-ethanone

(9e): White solid, $\mathrm{R}_{\mathrm{f}} \approx 0.50$ (petroleum ether, ethyl acetate 3:2), Melting point $110^{0} \mathrm{C},{ }^{1} \mathrm{H}$ NMR (600 MHz,CDCl 3 ): $\delta$ (in ppm) 1.21-1.26 $(1 \mathrm{H}, \mathrm{m}), 1.32-1.38(2 \mathrm{H}, \mathrm{m}), 1.42-1.45(1 \mathrm{H}, \mathrm{m})$, $1.61-1.62(1 \mathrm{H}, \mathrm{m}), 1.65-1.66(3 \mathrm{H}, \mathrm{m}), 1.70$ $(3 \mathrm{H}, \mathrm{s}), 1.86-1.87(1 \mathrm{H}, \mathrm{m}), 4.46(1 \mathrm{H}, \mathrm{d}, J=18)$, $4.75(1 \mathrm{H}, \mathrm{d}, J=18), 6.28(1 \mathrm{H}, \mathrm{d}, J=6), 6.82$ $(1 \mathrm{H}, \mathrm{d}, J=12), 7.08-7.13(3 \mathrm{H}, \mathrm{m}), 7.33-7.36$ $(2 \mathrm{H}, \mathrm{m}), 7.41-7.44(1 \mathrm{H}, \mathrm{m}), 7.72(2 \mathrm{H}, \mathrm{d}, J=6)$; ${ }^{13} \mathrm{C} \mathrm{NMR} \mathrm{(150} \mathrm{MHz,} \mathrm{CDCl}_{3}$ ): $\delta$ (in ppm) 22.66, $25.09,25.19,27.82,28.82,41.21,43.61$, $66.48,124.44,124.92,125.54,126.41$, 127.22, 127.69, 129.00, 132.59, 138.97, 169.06; HRMS (EI+): $\mathrm{m} / \mathrm{z}$ Calcd for $\mathrm{C}_{21} \mathrm{H}_{24} \mathrm{~N}_{2} \mathrm{O}_{3} \mathrm{~S}(\mathrm{M})^{+}$384.15076, Found: $\mathrm{m} / \mathrm{z}$ 384.1505. IR (in $\mathrm{cm}^{-1}$ ) 559.15, 578.64, 632.66, 690.79, 722.93, 748.91, 951.67, 1044.33, 1055.27, 1091.86, 1162.98, 1262.13, 1367.79, 1313.76, 1445.40, 1494.98, 1583.54, 1661.15, 2928.99, 2854.22, 3440.50 .

\section{1-(3-benzenesulf onyl-2-cyclohexyl-3,4- dihydro-2H-quinazolin-1-yl)-ethanone} (9f): White solid, $\mathrm{R}_{\mathrm{f}} \approx 0.50$ (petroleum ether, ethyl acetate $3: 2$ ), Melting point $120^{\circ} \mathrm{C},{ }^{1} \mathrm{H}$ NMR (600 MHz, $\left.\mathrm{CDCl}_{3}\right): \delta$ (in ppm) 0.98-1.10 $(4 \mathrm{H}, \mathrm{m}), 1.33-1.35(2 \mathrm{H}, \mathrm{m}), 1.47-1.50(2 \mathrm{H}, \mathrm{m})$, 1.57-1.61 (2H, m), $1.70(3 \mathrm{H}, \mathrm{s}), 1.85-1.87$ $(1 \mathrm{H}, \mathrm{m}), 4.43(1 \mathrm{H}, \mathrm{d}, J=18), 4.73(1 \mathrm{H}, \mathrm{d}, J=$ 18), $6.24(1 \mathrm{H}, \mathrm{d}, J=6), 6.82(1 \mathrm{H}, \mathrm{d}, J=6)$, 7.08-7.12 $(3 \mathrm{H}, \mathrm{m}), 7.33-7.36(2 \mathrm{H}, \mathrm{m}), 7.42$ $(1 \mathrm{H}, \mathrm{t}, J=6), 7.72(2 \mathrm{H}, \mathrm{d}, J=12) ;{ }^{13} \mathrm{C} \mathrm{NMR}$ $\left(150 \mathrm{MHz}, \mathrm{CDCl}_{3}\right.$ ): $\delta$ (in ppm) 22.43, 25.44, $25.88,27.95,28.85,29.66,37.85,43.42$, $66.92,125.58,126.47,127.21,127.62$, 129.01, 132.58, 134.79, 139.05, 169.34; HRMS (EI+): m/z Calcd for $\mathrm{C}_{22} \mathrm{H}_{26} \mathrm{~N}_{2} \mathrm{O}_{3} \mathrm{~S}(\mathrm{M})^{+}$ 
398.1664, Found: $\mathrm{m} / \mathrm{z}$ 398.16632. IR (in $\mathrm{cm}^{-}$ 1) 567.70, 578.64, 621.72, 647.70, 690.79, $718.82,744.81,930.13,964.66,1042.28$, 1091.86, 1152.04, 1169.47, 1175.97, $1260.08, \quad 1324.70, \quad 1352.74,1378.39$, $1447.45,1456,1499.43,1585.59,1658.76$, 2854.22, 2928.99, 3435.

\section{1-(2-Cyclopentyl-3-(4-fluoro-}

benzenesulf onyl)-3,4-dihydro-2Hquinazolin-1-yl)-ethanone (9j): White solid, $\mathrm{R}_{\mathrm{f}} \approx 0.40$ (petroleum ether, ethyl acetate 3:2), Melting point $150^{0} \mathrm{C},{ }^{1} \mathrm{H}$ NMR $\left(600 \mathrm{MHz} \mathrm{CDCl}_{3}\right): \delta$ (in ppm) 1.32-1.38 $(2 \mathrm{H}$, $\mathrm{m}), 1.42-1.44(1 \mathrm{H}, \mathrm{m}), 1.51(3 \mathrm{H}, \mathrm{s}), 1.62-1.67$ $(3 \mathrm{H}, \mathrm{m}), 1.77(2 \mathrm{H}, \mathrm{b} \mathrm{s}), 1.85-1.87(1 \mathrm{H}, \mathrm{m})$, $4.48(1 \mathrm{H}, \mathrm{d}, J=18), 4.68(1 \mathrm{H}, \mathrm{d}, J=18), 6.28$ $(1 \mathrm{H}, \mathrm{d}, J=12), 6.85(1 \mathrm{H}, \mathrm{d}, J=6), 7.01-7.04$ $(2 \mathrm{H}, \mathrm{m}), 7.08-7.16(3 \mathrm{H}, \mathrm{m}), 7.76-7.79(2 \mathrm{H}$, $\mathrm{m}) ;{ }^{13} \mathrm{C}$ NMR (150 $\mathrm{MHz}, \mathrm{CDCl}_{3}$ ): $\delta$ (in ppm) $22.49,25.06,25.18,27.81,28.84,41.29$, $43.61,66.44,116.06,116.21,124.53,124.91$, $125.71,126.48,127.38,130.50,130.56$, 135.03, 164.24, 165.94, 168.98; HRMS (EI+): $\mathrm{m} / \mathrm{z}$ Calcd for $\mathrm{C}_{21} \mathrm{H}_{24} \mathrm{~N} 2 \mathrm{O}_{3} \mathrm{~S}(\mathrm{M})+402.1413$, Found: $\mathrm{m} / \mathrm{z}$ 402.1415. IR (in $\mathrm{cm}^{-1}$ ) 568.96, 621.035, 680.82, 742.54, 808.11, 887.19, 925.76, 977.84, 1033.77, 1099.34, 1132.13, 1157.20, 1242.07, 1311.5, 1394.43, 1450.36, 1587.3, 1654.81, 2354.92, 2856.37, 2918.09, 2950.88, 3550.70, 3770.57 .

\section{Acknowledgment}

Moumita Chatterjee wishes to thank UGC, Suvankar Bera wishes to thank CSIR, Mohd Usman Mohd Siddique wishes to thank NIPER-KOLKATA for their respective fellowships. We would also like to thank the CSIR 'ORIGIN' CSC-0108 project for providing financial assistance towards this work. The authors would also like to thank the central instrumentation facilities of CSIR-IICB for recording the spectra.

\section{References}

1. ALAGARSAMY, V., RAJA SOLOMON, V. \& DHANABAL, K. 2007a. Synthesis and pharmacological evaluation of some 3phenyl-2-substituted-3H-quinazolin-4-one as analgesic, anti-inflammatory agents.
Bioorganic \& Medicinal Chemistry, 15 (1) 235241.

2. ALAGARSAMY, V., SOLOMON, V. R. \& MURUGAN, M. 2007b. Synthesis and pharmacological investigation of novel 4benzyl-1-substituted-4H-[1,2,4] triazolo[4,3a]quinazolin-5-ones as new class of $\mathrm{H} 1$ antihistaminic agents. Bioorganic \& Medicinal Chemistry, 15 (12) 4009-4015.

3. BRANDS, M., GRANDE, Y. C., ENDERMANN, R., GAHLMANN, R., KRÜGER, J. \& RADDATZ, S. 2003. Pyrimidinone antibiotics-heterocyclic analogues with improved antibacterial spectrum. Bioorganic \& Medicinal Chemistry Letters, 13 (16) 2641-2645.

4. CHEN, C.-J., SONG, B.-A., YANG, S., XU, G.-F., BHADURY, P. S., JIN, L.-H., HU, D.-Y., LI, Q.-Z., LIU, F., XUE, W., LU, P. \& CHEN, Z. 2007. Synthesis and antifungal activities of 5(3,4,5-trimethoxyphenyl)-2-sulfonyl-1,3,4thiadiazole and 5-(3,4,5-trimethoxyphenyl)2-sulfonyl-1,3,4-oxadiazole derivatives. Bioorganic \& Medicinal Chemistry, 15 (12) 3981-3989.

5. COMBY, F., LAGORCE, J. F., MOULARD, T., BUXERAUD, J. \& RABY, C. 1993. [Antibacterial sulfonamides, antiparasitic and antifungal derivatives of imidazole: evaluation of their antithyroid effects in the rat]. Vet Res, 24 (4) 316-26.

6. ELIOPOULOS, G. M. \& HUOVINEN, P. 2001. Resistance to TrimethoprimSulfamethoxazole. Clinical Infectious Diseases, 32 (11) 1608-1614.

7. GHORAB, M. M., RAGAB, F. A., HEIBA, H. I., YOUSSEF, H. A. \& GALA, M. 2010. Synthesis of some new pyrazole and pyrimidine derivatives carrying a sulfonamide moiety of expected antitumor activity and study of the synergistic effect of $\gamma$ irradiation. Arzneimittelforschung, 60(1) 48-55.

8. GIUSEPPE, C., PIERO, D. C., MADDALENA, G. \& CONCETTA, L. R. 2010. Tetrahydroquinazoline Derivatives by Aza 
Diels-Alder Reaction. HETEROCYCLES, 80 (2) 1457-1461.

9. HUOVINEN, P., SUNDSTROM, L., SWEDBERG, G. \& SKOLD, O. 1995. Trimethoprim and sulfonamide resistance. Antimicrob Agents Chemother, 39 (2) 279-89.

10. ISMAIL, M. A. H., BARKER, S., ABOU EL ELLA, D. A., ABOUZID, K. A. M., TOUBAR, R. A. \& TODD, M. H. 2006. Design and Synthesis of New Tetrazolyl- and Carboxybiphenylylmethyl-quinazolin-4-one

Derivatives as Angiotensin II AT1 Receptor Antagonists. Journal of Medicinal Chemistry, 49 (5) 1526-1535.

11. KAJINUMA, H.; KUZUYA, T. \& IDE, T. 1974. Effects of Hypoglycemic Sulfonamides on Glucagon and Insulin Secretion in Ducks and Dogs. Diabetes, 23 (5) 412-417.

12. KOMBU, R. S., MAILAVARAM, R. P., DEVALAPALLY, H., CHINNAPPA, P. M., DEVARAKONDA, R. K. \& AKKINEPALLY, R. R. 2008. Synthesis and Bronchodilator Studies of Some Novel 6-Alkyl/Aryl-1,2,4Triazino[4,3-c]Quinazolines. Open Med Chem J, 2 101-11.

13. KOREEDA, M. \& YANG, W. 1994. Chemistry of 1,2-Dithiins. Synthesis of the Potent Antibiotic Thiarubrine A. Journal of the American Chemical Society, 116 (23) 10793-10794.

14. KUMAR, K. S., GANGULY, S., VEERASAMY, R. \& DE CLERCQ, E. 2010. Synthesis, antiviral activity and cytotoxicity evaluation of Schiff bases of some 2-phenyl quinazoline- $4(3) \mathrm{H}$ ones. European Journal of Medicinal Chemistry, 45 (11) 5474-5479.

15. KUNES, J., BAZANT, J., POUR, M., WAISSER, K., SLOSAREK, M. \& JANOTA, J. 2000. Quinazoline derivatives with antitubercular activity. Farmaco, 55 (11-12) 725-9.

16. LOMBARDO, L. J., LEE, F. Y., CHEN, P., NORRIS, D., BARRISH, J. C., BEHNIA, K., CASTANEDA, S., CORNELIUS, L. A. M., DAS, J., DOWEYKO, A. M., FAIRCHILD, C., HUNT, J. T.,
INIGO, I., JOHNSTON, K., KAMATH, A., KAN, D., KLEI, H., MARATHE, P., PANG, S., PETERSON, R., PITT, S., SCHIEVEN, G. L., SCHMIDT, R. J., TOKARSKI, J., WEN, M.-L., WITYAK, J. \& BORZILLERI, R. M. 2004. Discovery of N-(2-Chloro-6-methyl- phenyl)2-(6-(4-(2-hydroxyethyl)- piperazin-1-yl)-2methylpyrimidin-4- ylamino)thiazole-5carboxamide (BMS-354825), a Dual Src/Abl Kinase Inhibitor with Potent Antitumor Activity in Preclinical Assays. Journal of Medicinal Chemistry, 47 (27) 6658-6661.

17. MARINI, A. M., MARESCA, A., AGGARWAL, M., ORLANDINI, E., NENCETTI, S., DA SETTIMO, F., SALERNO, S., SIMORINI, F., LA MOTTA, C., TALIANI, S., NUTI, E., SCOZZAFAVA, A., MCKENNA, R., ROSSELLO, A. \& SUPURAN, C. T. 2012. Tricyclic Sulfonamides Incorporating Benzothiopyrano[4,3-c]pyrazole and Pyridothiopyrano[4,3-c]pyrazole Effectively Inhibit $\alpha$ - and $\beta$-Carbonic Anhydrase: X-ray Crystallography and Solution Investigations on 15 Isoforms. Journal of Medicinal Chemistry, 55 (22) 9619-9629.

18. ÖZBEK, N., KATıRCiOĞLU, H., KARACAN, N. \& BAYKAL, T. 2007. Synthesis, characterization and antimicrobial activity of new aliphatic sulfonamide. Bioorganic \& Medicinal Chemistry, 15 (15) 5105-5109.

19. ROHINI, R., SHANKER, K., REDDY, P. M., HO, Y.-P. \& RAVINDER, V. 2009. Mono and bis-6-arylbenzimidazo[1,2-c]quinazolines: A new class of antimicrobial agents. European Journal of Medicinal Chemistry, 44 (8) 33303339.

20. TEMPERINI, C., CECCHI, A., SCOZZAFAVA, A. \& SUPURAN, C. T. 2008. Carbonic anhydrase inhibitors. Sulfonamide diuretics revisited-old leads for new applications? Organic \& Biomolecular Chemistry, 6 (14) $2499-2506$

21. VOLZHINA, O. N. \& YAKHONTOV, L. N. 1982. Quinazoline cardiovascular agents (review). Pharmaceutical Chemistry Journal, 16 (10) 734-741. 
22. XIE, M., UJJINAMATADA, R. K., SADOWSKA, M., LAPIDUS, R. G., EDELMAN, M. J. \& HOSMANE, R. S. 2010. A novel, broadspectrum anticancer compound containing the imidazo[4,5-e][1,3]diazepine ring system. Bioorganic \& Medicinal Chemistry Letters, 20 (15) 4386-4389. 